\title{
A Performance Evaluation of Signal Filtering with different Adaptive Algorithms on Doppler-Angular Adaptive Generalized Sidelobe Canceler (GSC)
}

\author{
Samer Emad Eldin \\ Radar Department \\ Military Technical College, \\ Cairo, Egypt \\ Hossam Eldin Abu Bakr \\ Radar Department \\ Military Technical College, \\ Cairo, Egypt
}

\author{
Hazem Zakarya Kamel \\ Radar Department \\ Military Technical College, \\ Cairo, Egypt \\ Hossam Sabry Tork \\ Radar Department \\ Military Technical College, \\ Cairo, Egypt
}

\begin{abstract}
In modern radar signal processing the Space-Time Adaptive (STAP) filtering establishes a significant part of statistical signal processing. Whenever there is requirement to process signals that result from operation in an environment of unknown statistics, the use of an adaptive filter offers an attractive solution to the problem as it usually provides a significant improvement in performance over the use of a fixed filter designed by conventional methods.

In this paper we study the use of three different adapting algorithms for adaptive generalized sidelobe canceler of interference signal suppression which intercepted by a uniform linear phased array antenna. Results are plotted, discussed and compared to each other. The paper is divided into four main sections. The problem is defined in the introduction section. In the next section, the adaptive spatial-temporal processor is depicted as well the autocorrelation matrix $R$ has been driven. Next, the filtering using the normalized least mean-square (NLMS) algorithm was discussed. Also, we study the use of normalized fractionally low-order moment algorithm (NFLOM) for adapting of the same uniform linear array antenna and it was explained in section 6. It was compared to the NLMS algorithm for rate of convergenc, minimum mean-square error and angular-doppler beam pattern. In the last section, The recursive least-square (RLS) algorithm was implemented to get the adaptive filtering weight vector and compared to previouse algorithms. Finally, we've concluded all the results discussed in each algorithm.
\end{abstract}

Keywords: STAP, NLMS, NFLOM, RLS.

\section{INTRODUCTION}

Appling a temporal filtering and spatial beamforming combination on the signals intercepted by phased-array radar systems are denoted as Space-time adaptive processing (STAP). It utilizes an antenna composed of array of elements afterwards to obtain multiple pulses processed coherently it is passed through tapped-delay-lines, therefore it provides a greater capability to deduce the influence of jammers and clutters on the system while preserving desired signal target [1]. A thoroughly researching on STAP was employed and it is revealed to deliver gains of remarkable performance in target detection and interference suppression [2]. Various
STAP processors manage frequent situations clutters, noises and jammers are complex Gaussian, that drives to solutions of tractable mathematics [4, 24, 25].one of significant solutions is the adaptive beamforming.

At different scenarios, ranging from radar and wireless communications to sonar is found to be applications for the adaptive beamforming technique. It is founded on a system where, an array of elements weights at attached filters are adjusted to achieve a selective spatial and spectral. Fig illustrates the desired signal intercepted by a beamformer using $\mathrm{M}$ sensor elements.

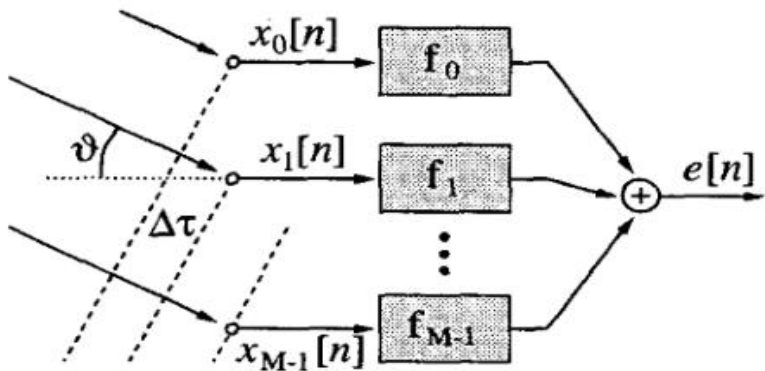

Fig. 1. Beamforming using $\mathrm{M}$ elements receives desired signal impinging from (DOA) angle

Attention here to linearly constrained minimum variance (LCMV) beamformer is directed, because of its efficiency to be employed as a generalized sidelobe canceller (GSC) which has an unconstrained optimization problem as an alternative of a constrained one. Instead of employing beamforming, techniques of adaptive filtering are applied at the GSC adaptive process structure to perform a deep interference nulling and high resolution by using multiple sensors placed in arrays and filter weights.

In next section the optimum processor for wideband interference cancellation is revised in terms of the standard direct processor system with a covariance matrix is known. The corresponding full-dimension generalized sidelobe canceller (GSLC) structure [3, 4] of the processor is derived in Section 3. Two different methods referring to commonly 
used algorithmic families have been implemented for the adaptively estimated MSE optimized parameters based on the available set of data. On the basis of steepest descent stochastic approximation method which is known as the Least Mean Squared (LMS) family the first approach is utilized in Section 6. The latter is the Recursive Least Squares (RLS) approach which is referred as the GaussNewton stochastic approximation.

The equivalence between the the Constrained RLS (CRLS) and GSC-RLS was verified by Werner [5] leads to the GSC form by employing the Recursive Least Squares (RLS) algorithm, which is called GSC-RLS, was derived in Section 8.

This paper is organized as follows. Section 2 describes Space-Time Adaptive Processing (STAP). Section 3 presents GSLC Processor Form. Section 4 presents Design of Blocking Matrix. Section 5 presents GSLC adaptation using the NLMS.

Section 6 presents Simulation results of NLMS-GSC and NFLOM-GSC. Section 7 presents GSLC adaptation using proposed recursive least square (RLS) algorithm for STAP. Section 8 presents Simulation Results of RLS-GSC algorithm performance.

\section{Space-Time Adaptive Processing (STAP)}

Signals intercepted by the antenna array are represented as 3D data of collected coherent processing interval (CPI) waveform at array elements. The data at the only desired range gate is then handled, which is represented as a CPI data slice. This part of CPI data contains a snapshot of NxM space-time data that consist of different components related to the information from the ith $(i=1,2, \ldots, N)$ element and $j$ th $(\mathrm{j}=1,2, \ldots, \mathrm{M})$ tap. Therefore, this two dimensional data structure of space-time comprises of tap information and space element. The samples is then stacked in a column to create vector $U$ of $\mathrm{NM} \times 1$-dimensional. If a range cell of interest contains the target, then the received signal is formed of elements due to the target, white Gaussian noise, and the field interference signals (jammers sources and clutter):

$$
U=U_{t}+U_{j}+U_{c}+U_{n}
$$

If the target does not exist, then the sample received will only consist of white noise, and interference. Then $U$ vector is:

$$
U=U_{j}+U_{c}+U_{n}
$$

The covariance matrix of the input will be

$$
R=E\left\{U U^{H}\right\}
$$

The $N M \times 1$-dimensional space-time steering vector

$\mathbf{v}\left(\mathrm{v}_{\mathrm{t}}, \mathrm{w}_{\mathrm{t}}\right)=\mathrm{b}\left(\mathrm{w}_{\mathrm{t}}\right) \otimes \mathrm{a}\left(\mathrm{v}_{\mathrm{t}}\right)$

(4)
Where $b\left(w_{t}\right)$ is the steering temporal vector of $M \times 1$ dimension at the frequency $w_{t}$ of target signal and $a\left(v_{t}\right)$ represent the target spatial steering vector of $N \times 1$-dimension at the direction delivered by spatial frequency of the targt $v_{t}$. The operator of Kronecker product is noted by (.) $\otimes$ (.). Conveniencly, the steering vector in the space-time direction of interest is normalized and set to be

$$
S=\frac{v\left(v_{t}, w_{t}\right)}{\sqrt{v^{H}\left(v_{t}, w_{t}\right) \boldsymbol{v}\left(v_{t}, w_{t}\right)}}
$$

The SINR unconstrained optimization provides vector $w_{o}$ that contains the optimum weights will be $[6,7]$

$$
w_{o}=R^{-1} S
$$

Considering the weight vector in (6) is providing target detection by utilizing a constrained optimization under assumption of the joint Gaussian. To maximize the output SINR, the vector of weights under optimal constrains that maintain the response of the space-time snapshot at targetdirection normalized, was originally given in $[8,3]$ as following:

$$
w_{o}=\frac{R^{-1} s}{s^{H} R^{-1} s}
$$

The input data is applied to this weight vector, the output $y$ is then formed as

$$
y=w_{o}{ }^{H} U=\frac{s^{H} R^{-1} U}{s^{H} R^{-1} s}
$$

and the output SINR be

$$
\xi=\frac{|\alpha|^{2}}{P_{n}}=\frac{|\alpha|^{2}}{w_{o}{ }^{H} R w_{o}}=|\alpha|^{2} s^{H} R^{-1} S
$$

Where, $|\alpha|^{2}$ is a target waveform output power, and $P_{n}$ is the processor output power of the noise and it is given by

$$
P_{n}=\frac{1}{s^{H} R^{-1} s}
$$

As stated above the constrained optimization leads to the weight vector in (7). This weight vector is resulting in a beam, which is located in a specific direction of looking, spatially and temporally that is provided by the vector of steering s. That spatial-temporal processor structure is showed in Figure 2. 


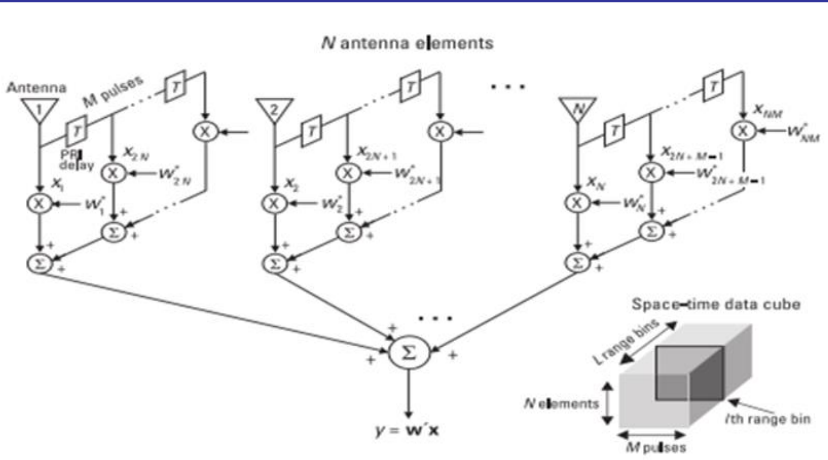

Fig. 2. Space-Time adaptive processor [9]

The adaptive tapped delay line architecture required to perform STAP is shown in Fi. The weights w (adaptive vector), are employed at every one tap of the taps conducted at each spatial channel prior to the Final beamforming summation. Thus, $N \times M$ is the total adaptive coefficients number to be considered where $N$ spatial Channels with $M$ taps per channel are utilized. Note; $\tau$ is the taps in-between delay of time.

The minimum variance distortionless response (MVDR) scheme is usually applied for $\mathrm{W}$ the adaptation vector of weights in the Gaussian clutter environment for the output signal of second-order-moment minimization which is subjected to steering constraints

$$
\min _{W} E\left\{|y(t)|^{2}\right\}, \text { subjected to } s^{H} W=h,
$$

Where the expectation operator used is $\mathrm{E}\{\cdot\}, E\left\{|y(t)|^{2}\right\}=W^{H}$ $R u u W$, and Ruu is the input vector U covariance matrix. $S$ is the matrix of linear constraints set and $h$ is the vector of desired response.

Assume that, a constraint of a simple point can be selected as $S=b\left(\omega_{t}\right) \otimes a\left(\Theta_{t}\right)$ and $\mathrm{h}=1$, where a response of a unity gain is applied at the frequency Doppler $f_{t}$ and at the position of the target $\Theta_{t}$. Assuming a full rank for the $\mathrm{R}$ (matrix of covariance)then the constrained minimization problem (11) has a well-known optimal solution [10]:

$$
w_{o}=R^{-1} s\left(s^{H} R^{-1} s\right)^{-1} h .
$$

Implementing (12) directly requires the knowledge of the input vector covariance matrix of the elements array and the sample-matrix-inversion (SMI) scheme is usually applied [2]. Instead, the decomposition of the vector of weights $W_{\mathrm{o}}$ into two modules orthogonally is employed: one is a vector of unconstrained adaptive weights $W a$ and the other is a fixed beamformer $W_{q}$. This decomposing type is recognized as the generalized sidelobe canceller (GSLC) where $W a$ could be adapted in iterative way by any least-square adaptive methods. Next section will point out this GSLC processor form and it's relying on the output signal to the desired one mean-square error optimization.

\section{III.GSLC PROCESSOR FORM}

The processor structure of the STAP in a direct way is reformed to a GSLC processor type. A GSLC leads to an unconstrained weight vector, where processor form of the STAP is transformed to a Wiener filter form. The GSLC processor structure delivers a deeper insight into problems of reducing dimension otherwise these problems appears to be possible. Considering operator " $T$ " of the unitary matrix of dimension $N M \times N M$ as transformation operator of the detection priorly data. This operator structure is decomposed as follows:

$$
\text { IV. } T=\left[\begin{array}{l}
s^{H} \\
B_{m}
\end{array}\right]
$$

Where "s" is the conventional space-time beamformer of a dimension $N M \times 1$ that defined in (5) and $B_{\mathrm{m}}$ is signal $C \times$ $N M$ dimensional blocking matrix with full-raw rank which map the data input " $u$ " into the null space of space-time beamformer " $s$ ", where $C=(N M-1)$ Hence,

$$
B_{m} s=0
$$

The waveforms intercepted at the spatio-temporal direction of interest is effectively blocked by the matrix $B_{\mathrm{m}}$. Considering matrix $B_{\mathrm{m}}$ of a full-row rank to be a valid matrix of signal-blocking which fulfills (14) and provide $\mathrm{T}$ to be an invertible. such reformulation results in an output SINR is the same as the direct-form of the original processor of detection.

\section{DESIGN OF BLOCKING MATRIX}

The Blocking Matrix (BM) structure, $B_{\mathrm{m}}$ acts significantly in the structure of the GSC, the computational complexity depends on its choice and in many cases, it strengths the overall system numerical stabilities [11]. Determining of a blocking matrix $B_{\mathrm{m}}$ and the proper quiescent vector $\mathbf{s}$ which satisfies the constraints required for the GSC structure. The suitable BM design can be acquired through employing one of two algorithms for directly obtaining an orthonormal $B_{\mathrm{m}}$ from the desired normalized steering vector s. The matrix Singular Value Decomposition (SVD) is invoked by the first algorithm which is more accurate. The SVD approach may be employed for any constraint matrix $\mathrm{C}$ and is not limited to broadside limitations case. Hence, it is a general strategy for finding BM [12]. The other approach utilizes the QR-decomposition, and is more rapidly. Each of these approaches are explained as well as is derived in depth in numerous numerical and linear algebra texts $[13,14]$.

\section{Algorithm 1:}

$[\mathrm{U}, \mathrm{S}, \mathrm{V}]=\operatorname{svd}\left(\mathrm{s}^{\mathrm{T}}\right)$

$B_{\mathrm{m}}=[\mathrm{V}(:, 2: \mathrm{N})]^{\mathrm{T}}$

Algorithm 2:

$[\mathrm{Q}, \mathrm{R}]=$ qr $(\mathrm{s})$

$B_{\mathrm{m}}=\left[\mathrm{Q}^{2}(:, 2: \mathrm{N})\right]^{\mathrm{H}}$ 
A third algorithm has been proposed to formulate the $\mathrm{BM}$ is the cascaded columns of differencing (CCD) [15].

\section{a) Cascaded-Columns Differencing (CCD)}

The proposed algorithm of CCD is offered to get the derivative constraints blocking matrix. The durability on front of the direction of interest signal errors is increased by these constraints through enlarging the targeted constraints' angular range. As the order of the derivative constraints is getting higher, the wider the beam pointing in the desired direction [15].At CCD approach, a blocking matrix is designed by $\mathrm{S}$ cascaded columns differencing operations of as in Fig [12].

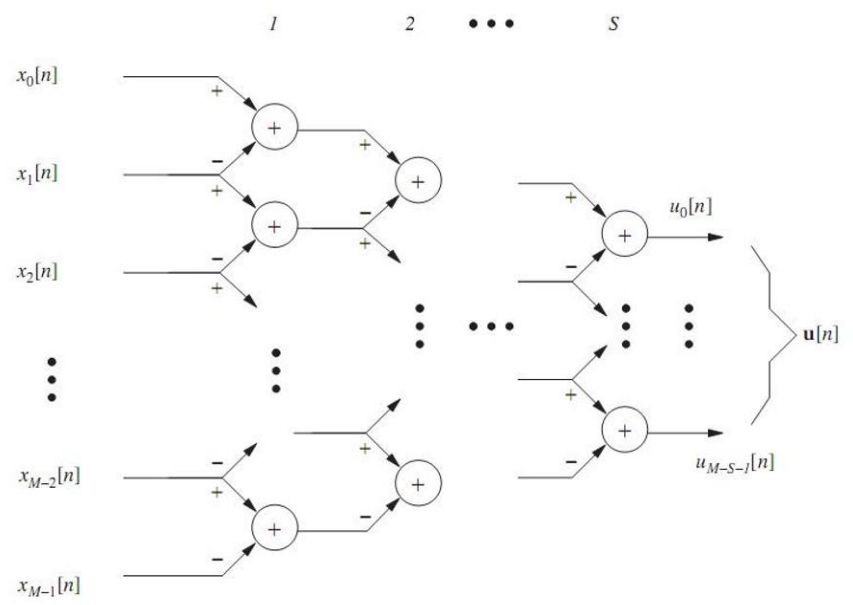

Fig. 3. S columns form the CCD to obtain the BM [12]

The BM is formulated in a matrix form as follows:

$$
\widetilde{B}=B_{M} \cdot B_{M-1} \ldots B_{M-S+1}
$$

Where we have

$$
B_{i}=\left[\begin{array}{ccccc}
1 & -1 & \cdots & & 0 \\
& \vdots & \ddots & & \vdots \\
& 0 & \cdots & 1 & -1
\end{array}\right]^{H}
$$

By $\mathrm{i}=\mathrm{M}, \mathrm{M}-1 . . \mathrm{M}-\mathrm{S}-1$. It's clear that, if a waveform returns from a wide side, it won't be capable of passing this $\mathrm{BM}$. The BM in broadside forms a zero response and with increasing $\mathrm{S}$ a lobe of wider width is obtained [12].

Much of the time, it may be easy to construct a nonorthonormal signal blocking matrix and the orthonormalization can be provided by implementing the procedure of Gram-Schmidt. One case of this is a system, which the angle and time array manifold is set by utilizing a primary separate vector steering of space-time. Accordingly, in regard to the array, the s normalized steering vector is engaged to a broadside point. In such cases, an appropriate input signals difference is simply taken to provide the nonorthonormal matrix of signal blocking which is the same as the array configuration for arrays of narrowband offered in [16].
The radar incoming signal $\mathrm{u}$ is transformed using the operator $\mathrm{T}$ in (13) incomes a vector $\tilde{u}$, which takes the form

$$
\tilde{u}=T u=\left[\begin{array}{l}
s^{H} u \\
B_{m} u
\end{array}\right]=\left[\begin{array}{l}
v \\
b
\end{array}\right]
$$

Where $\mathrm{v}$ is termed as the scalar-valued beamformed output. Here, also the vector $b$ with dimension (NM-1) is denoted as data vector of the noise subspace. Then vector $\tilde{u}$ that provide the data transformed has its related covariance matrix $\mathrm{R}_{\widetilde{\mathrm{u}}}$, and it is given as:

$$
R_{\widetilde{u}}=T R T^{H}=\left[\begin{array}{cc}
\sigma_{v}^{2} & r_{b v}^{H} \\
r_{b v}^{H} & R_{b}
\end{array}\right]
$$

Where $R_{b}$ is the covariance of the noise subspace which is of dimension $\mathrm{C} \times \mathrm{C}$ is given by

$$
R_{b}=E\left[b b^{H}\right]=B_{m} R B_{m}^{H}
$$

The Cx1-dimensional vector $r_{b v}$ is the output of the beamformer cross-correlation to data vector of the interference subspace and is expressed by

$$
r_{b v}=E\left[b v^{*}\right]=B_{m} R s
$$

Where the operator of the complex conjugate is represented by "*". The beamformer output variance is denoted as the scalar $\sigma_{d}^{2}$ in (18) that is set to be

$$
\sigma_{v}^{2}=s^{H} R s
$$

Next, let $s$ (the vector of steering) is transformed by the operator $T$. The unit transformed, steering vector $e_{l}$, is given by this operation and it is given by

$$
e_{1}=T s=\left[\begin{array}{c}
1 \\
0 \\
\cdot \\
\vdots \\
0
\end{array}\right]
$$

Then, these transformed coordinates has the optimal weight vector which is given by (as $\left.\mathrm{w}_{0}\right)$ [17]:

$$
w_{g S c}=\frac{R_{\widetilde{u}}^{-1} e_{1}}{e_{1}^{H} R_{\widetilde{u}}^{-1} e_{1}}
$$


Where, $R_{\widetilde{u}}$ is that covariance matrix expressed in (18). The GSLC processor structure showed in Fig $[18,19]$ is normally derived as the separating of the matrix operator $T$ is applied, this processor leads for the upper branch a unity fixed weight and a $C=N M-1$ dimensional vector $w$ as an adaptive weight vector in the lower branch. Regarding to the configuration shown in Fig the vector $w$ in (23) with optimal value is provided by the Wiener solution. Then the optimum weight w defined as

$$
w=R_{b}^{-1} r_{b v}
$$

Where, $R_{b}$ and $r_{b v}$ are computed in (19) and (20), respectively.

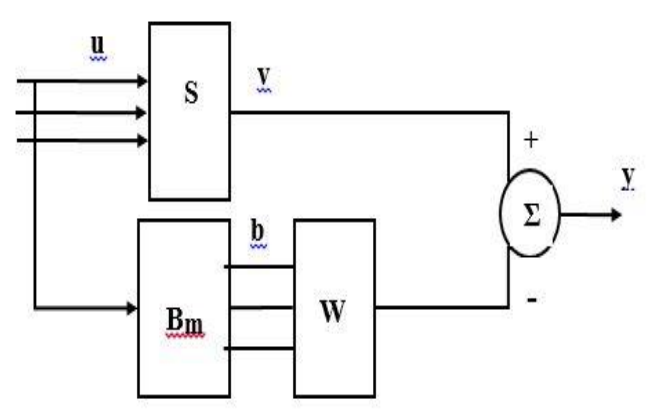

Fig. 4. full dimention GSLC processor

The GSLC structure processor employs the $N \times M$ weight vector in (23) by applying the splitting given in (17). The GSLC performance at the steady state in the operation mode of adaptive data is identical to the direct-form processor performance, but a lower dimension vector of the adaptive weights $w$ is used by the GSLC. Therefore, a reduction of the computations required for the weight vector updating is obtained and hence, the canonical form of processor can be the GSLC processor.

Employing the weight vectors defined in (7) and (23) on the data arrays, leads an identical output, and therefor utilizing (17) and (23), it may be defined as follows:

$$
\begin{gathered}
y=w_{\circ}^{H} u=w_{g s c}^{H} \tilde{u}=\left(S^{H}-\right. \\
\left.w^{H} B_{m}\right) u
\end{gathered}
$$

Substituting (24) into (25) and (10) is used to the evaluate the mean-square value of $y$; the minimum noise power of the output is obtained as follows:

$$
P_{n}=\frac{1}{S^{H} R^{-1} S}=\sigma_{d}^{2}-r_{b v}^{H} R_{b}^{-1} r_{b v}
$$

Considering the filters $\mathrm{S}$ and $\mathrm{B}_{\mathrm{m}}$ definitions, the output SINR stated by (9) can be reformulated as follows:

$$
\xi=\frac{|\alpha|^{2}}{\sigma_{d}^{2}-r_{b v}^{H} R_{b}^{-1} r_{b v}}
$$

In the form of eigenvectors and eigenvalues, the covariance matrix $R_{b}$ is represented as :

$$
R_{b}=U \Lambda U^{H}
$$

where the eigenvectors $\left\{v_{i}\right\}_{i=1}^{N}$ forms a unitary matrix of dimension $C \times C$ denoted by $U$ and the related eigenvalues $\left\{\lambda_{i}\right\}_{i=1}^{N}$ formed the diagonal values of a diagonal matrix represented by $\Lambda$. Transforming the noise process $b$, which is implicitly defined in $u=T u=\left[\begin{array}{ll}s^{H} u \\ B_{m} u\end{array}\right]=\left[\begin{array}{l}v \\ b\end{array}\right]$

(17) to a principal coordinate

process $p$ gives:

$$
p=U^{H} b
$$

The covariance matrix of normal transformed components, $R_{p}$ cross-correlation vector $r_{b d}$ and $W_{N}$ the Wiener filter are defined now as follows:

$$
\begin{aligned}
& R_{p}=E\left[p p^{H}\right]=U^{H} R_{b} U=\Lambda \\
& r_{p v}=E\left[b v^{*}\right]=U^{H} r_{b v} \\
& w_{N}=R_{b}^{-1} r_{b v}=U^{H} w
\end{aligned}
$$

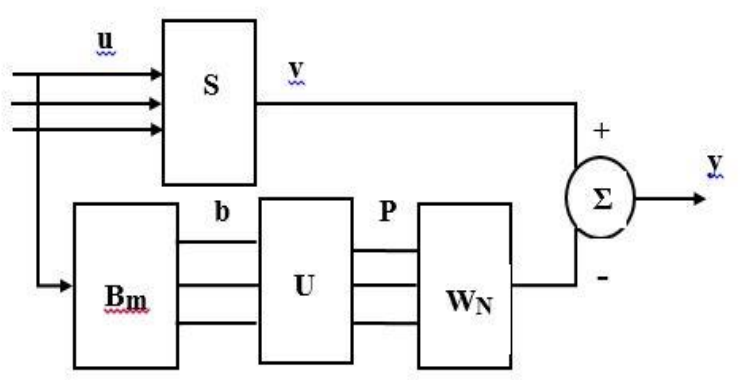

\section{Fig. 5. Principal coordinates form of full- dimension GSLC processor}

These normal coordinates with its GSLC form is illustrated in Error! Reference source not found.. In the adaptive data processing mode, the characteristics of the steady state of both array processors are the same, however they may perform differently. The GSLC output array $y$ defined by:

$$
\begin{gathered}
y=\left(S^{H}-w_{N}^{H} U^{H} B_{m}\right) u= \\
\left(S^{H}-w^{H} B_{m}\right)
\end{gathered}
$$


Annotate that the minimum noise power at the output.

$$
P_{n}=\sigma_{d}^{2}-r_{p v}^{H} R_{p}^{-1} r_{p v}=\sigma_{d}^{2}-r_{p v}^{H} R_{b}^{-1} r_{b v}
$$

and that is the SINR,

$$
\xi=\frac{|\alpha|^{2}}{\sigma_{v}^{2}-r_{p v}^{H} R_{p}^{-1} r_{p v}}=\frac{|\alpha|^{2}}{\sigma_{v}^{2}-r_{b v}^{H} R_{b}^{-1} r_{b v}}
$$

is conserved by the operator $U$.

\section{IX.GSLC ADAPTATION USING THE NLMS}

The structure Displayed in Fig [20] involves of two channels that is identified as lower channel and upper channel. The upper channel usually denoted as the fixed beam former and an adaptive section along with blocking matrix form the lower channel.

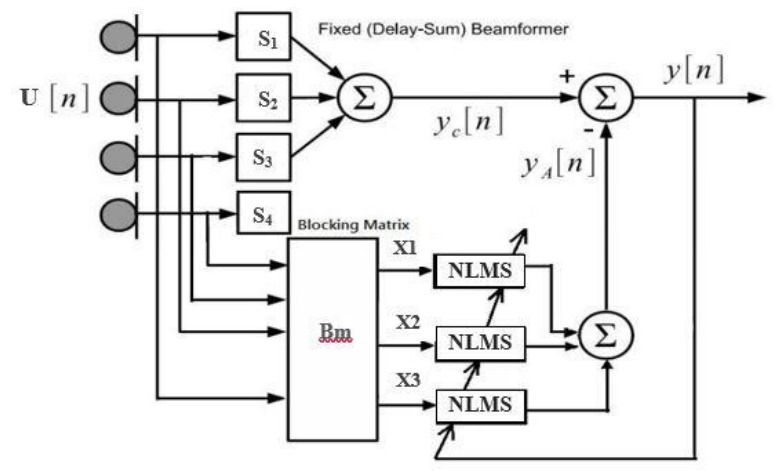

Fig. 6. Adaptive Generalized Side-lobe Canceller Structure

A set of filters, at the adaptive part, is combined to adaptively minimize the output power. The Blocking Matrix $(\mathrm{BM})$ is employed to have the wanted waveform be eliminated at the second path, confirming that it is the noise power that required to be minimized. Then at the adaptive path the weight vector of the filters is updated by applying the algorithm of NLMS having $y[n]$ as signal reference, the weights update equation is like as below:

$$
W_{a}(t+1)=W_{a}(t)+\mu a \frac{x(t) e^{*}(t)}{x^{H}(t) x(t)+\delta}
$$

Where $\mathrm{x}(t)=x(t)=B_{m}^{H} u(t)$,

and $e(t)=y(t)=\left[\boldsymbol{S}-B_{m} W_{a}\right]^{H} u(t)$ define the error signal. The converging rate is controlled by $\mu a$ as a size of step. The numerical instability when the inputs are small is prevented by the regularization parameter $\delta[10]$.

The NLMS algorithm in (36) is equivalent to:

$$
W(0)=\boldsymbol{S}=s\left(s^{H} s\right)^{-1} h
$$

$$
W(t+1)=G\left[W(t)-\mu \frac{u(t) y^{*}(t)}{u^{H}(t) G u(t)+\delta}\right]+
$$

$\boldsymbol{S}$

Where the step size is defined as $\mu$ and the conjugate denoted by the superscript $*$

$$
G=I-s\left(s^{H} s\right)^{-1} s^{H}
$$

\section{X.GSLC ADAPTATION USING NORMALIZED FRACTIONALLY-LOWER-ORDER MOMENT (NFLOM) ALGORITHM}

Performance loss is suffered by the conventional STAP algorithm under the severe, impulsive clutter environment as a result of 2 causes: first is the receiving of snapshots where the outliers' probability is high; second is the snapshot covariance matrix with large eigenvalue spread. The FLOM approach is An algorithm to overcome these difficulties. Rather than the STAP output's variance minimization this approach reduces the $\mathrm{p}^{\text {th }}$ order-moment $[21,22]$.

$$
\min _{W} E\left\{|y(t)|^{p}\right\} \text {, subjected to }{ }^{H} W=h,
$$

The resolving of optimum weights where the cost function is minimized has no closed-form, but a gradient descent algorithm is presented. The same as the NLMS method, the iterative adaptation is applied to the NFLOM algorithm as

$$
W(0)=0,
$$

$$
W a(t+1)=W a(t)+\mu a \frac{|e(t)|^{p-2} e^{*}(t) x(t)}{\sum_{i}\left|x_{i}(t)\right|^{p}+\delta}
$$

Equivalently, the algorithm in $W a t+1$ ? $=W a(t)+$ $\left.\mu a \frac{|e(t)|^{p-2} e^{*}(t) x(t)}{\sum_{i}\left|x_{i}(t)\right|^{p}+\delta}, \quad\right)$ is the same as:

Equivalently, the algorithm in $W a t+1$ ? $=W a(t)+$ $\left.\mu a \frac{|e(t)|^{p-2} e^{*}(t) x(t)}{\sum_{i}\left|x_{i}(t)\right|^{p}+\delta}, \quad\right)$ is the same as:

$$
W(0)=S=s\left(s^{H} s\right)^{-1} h
$$

$$
\begin{array}{r}
W(t+1)=G[W(t)- \\
\left.\mu \frac{|y(t)|^{p-2} y^{*}(t) u(t)}{\sum_{i}\left|x_{i}(t)\right|^{p}+\delta}\right]+\boldsymbol{S}
\end{array}
$$

Where $x_{i}(t)$ are the output of block matrix elements $\mathrm{x}(\mathrm{t})=\mathrm{B}_{\mathrm{m}}{ }^{\mathrm{H}} \mathrm{u}(\mathrm{t})$. the rest of parameters are similar to the 
NLMS algorithm parameters in $W t+1$ ? $=$
$G\left[W(t)-\mu \frac{u(t) y^{*}(t)}{u^{H}(t) G u(t)+\delta}\right]+S$

We can have the NLMS algorithm by reducing the NFLOM algorithm using $\mathrm{p}=2$ and when $\mathrm{p}=1$ the NFLOM algorithm is reduced to the algorithm of normalized sign (NSA).

\section{XI.SIMULATION RESULTS OF GSLC ADAPTATION USING NFLOM ALGORITHM}

The offered NLMS-GSC and NFLOM-GSC algorithms' evaluation is demonstrated by two typical adaptive spacetime processor examples. The first experiment simulation scenario is adopted as a stationary narrowband target signal, $\mathrm{s}_{\mathrm{T}}(\mathrm{n})$, had a $0 \mathrm{~dB}$ power relative to noise power at background . It has $0 \circ$ as arrival angle(AoA) relative to array antenna elements. The target Doppler frequency that is normalized is fixed at 0.3 . The signal of the target is diminished by two stationary narrowband source of jamming $\mathrm{s}_{\mathrm{j} 1}(\mathrm{n}) \mathrm{s}_{\mathrm{j} 2}(\mathrm{n})$, in different intercepted angle.

Noise signal of background $(\eta(n))$, is Gaussian white distributed with zero mean. Also, Clutters were distributed from $-180^{\circ}$ to $180^{\circ}$ at different DoAs that have Doppler frequencies that count on their DoAs. An average power ($30 \mathrm{~dB})$ is the Rayleigh distributed Clutter's envelop.

$\mathrm{u}(\mathrm{n})=\eta(\mathrm{n})+\mathrm{s}_{\mathrm{j} 1}(\mathrm{n})+\mathrm{s}_{\mathrm{j} 2}(\mathrm{n})+\mathrm{s}_{\mathrm{T}}(\mathrm{n})$.

The parameter $\theta$ is referred as the plane waveform incoming angle (with respect to the broadside) and $\mathrm{f}$ denotes the normalized frequency of the received signal. The equally spaced linear array elements of $\mathrm{N}=15$ form the input channels of the adaptive space-time processor. The distance between every two elements are half of the wavelength related to $f_{\max }$, which is navigated to the target waveform direction. For each antenna element 10-delay elements are assigned to it. $\mathrm{M}=10$ is the coherent pulse interval (CPI) and a fixed range gate were employed for the space-time adaptive processing.

The mean square error e(n) of the actual target signal and the STAP output, $\quad \mathrm{s}_{\mathrm{T}}(\mathrm{n}), \quad$ (i.e., $\left.\min \mathrm{E}\left[\left|\mathrm{s}_{\mathrm{T}}(\mathrm{n})-\mathrm{e}(\mathrm{n})\right|^{2}\right]\right)$ was computed at every case through estimated instantaneous squared errors' averaging over a window exponentially decayed with instants of time memory equal. The NLMSGSC and NFLOM-GSC algorithms have the learning curves that are showed in Fig. The offered approach (afterwards the convergence) has beam patterns are illustrated in Fig.

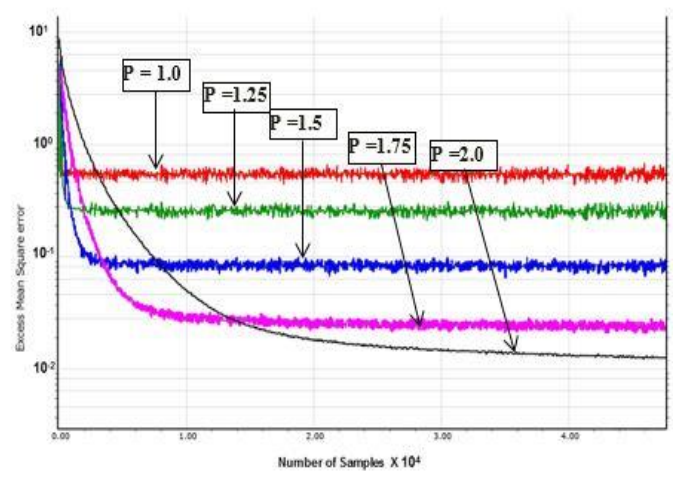

Fig. 7. Excess MSE for $\operatorname{NLMS}(p=2)$ and $\operatorname{NFLOM}(p=1.0, \ldots, 1.75)$

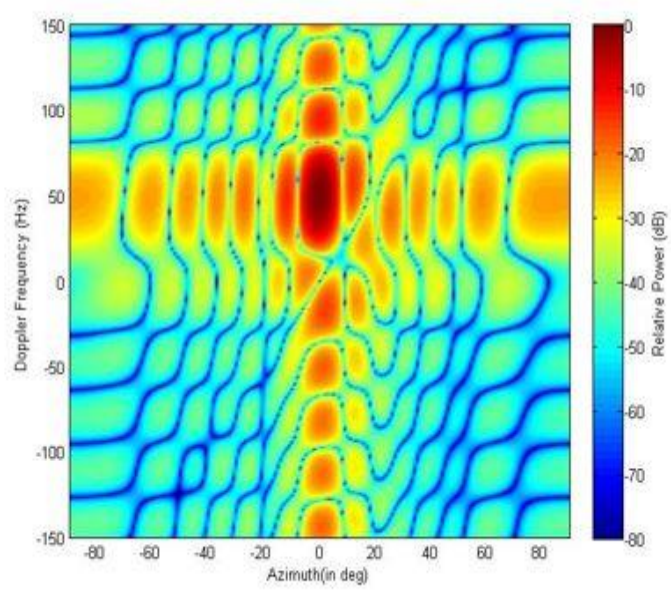

Fig. 8. Beam pattern of the NLMS algorithm $(\mathrm{p}=2)$

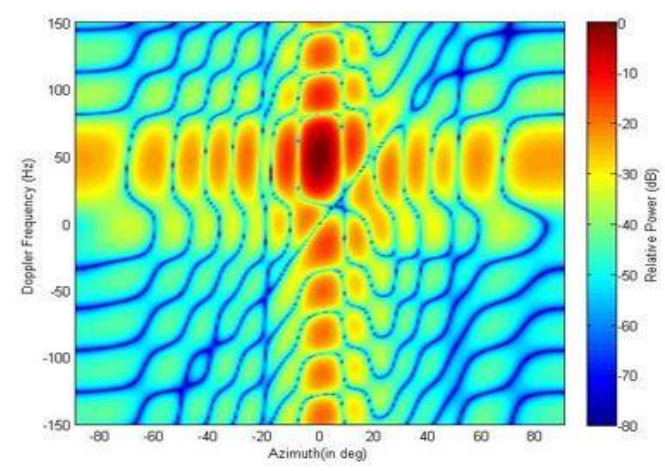

Fig. 9. Bemapattern of) NFLOM with $\mathrm{p}=1.7$ : maintained deep nulls at jammer locations

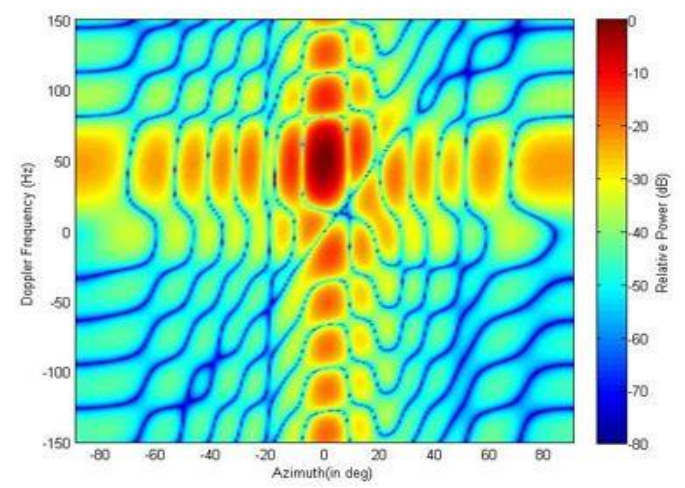

Fig. 10. Normalized Radiation Pattern with and without adaptation 16 element linear array and jammers at $(-35,15 \mathrm{deg})$

\section{GSLC ADAPTATION USING PROPOSED RECURSIVE LEAST SQUARE (RLS) ALGORITHM FOR STAP}

The main issue here at the space-time adaptive GSLC as discussed earlier is the way of obtaining of the variable weight vector $W_{a}$. GSLC-RLS method is one of the more well-known approaches in resolving this problem. The 
GSLC-RLS method is built on the structure of the GSLC and the variable weight vector $W_{a}$ is adjusted by applying the Recursive Least Squares (RLS) method. The GSC-RLS method is described in details later.

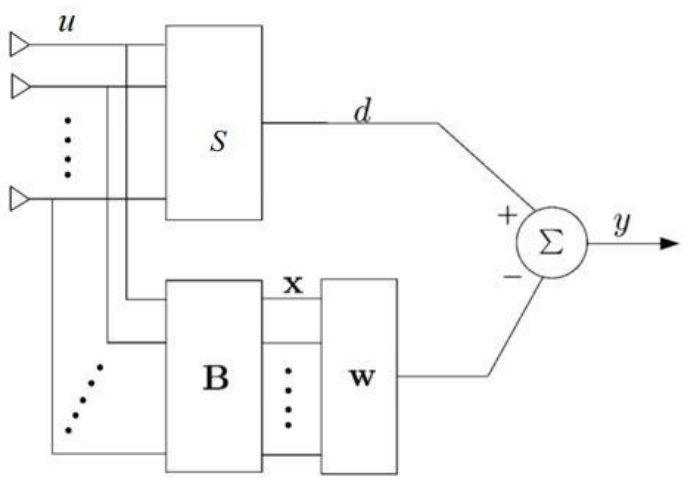

Fig. 11. STAP with GSC architecture

Using the RLS method which is a recursive solution of the least squares, the adjusted weight vector of GSLC can be adapted as $[5,10]$

$$
x(n)=B_{m}^{H} u(n)
$$

$\mathrm{B}_{\mathrm{m}}$ is the matrix block of the signal, that is consisted of any set of basis that are orthogonal for the directing array of the target $\mathrm{u}_{\mathrm{t}}$. Therefore, we have

$$
\mathrm{B}_{\mathrm{m}} \mathrm{u}_{\mathrm{t}}=0 \text {, }
$$

In general, the blocking matrix can be straightly acquired by utilizing the $\mathrm{QR}$ decomposition or the singular value decomposition (SVD) algorithms [42] as discussed in earlier section.

$$
\begin{aligned}
& d(n)=S^{H} u(n) \\
& e(n)=d(n)-w_{a}^{H}(n-1) x(n) \\
& b(n)=\left[S-B_{m} W_{a}\right]^{H} u(n) \\
& k(n)=\frac{P(n-1) x(n)}{\lambda+x(n)^{H} P(n-1) x(n)} \\
& W_{a}(n)=W_{a}(n-1)+k(n) e^{*}(n) \\
& \quad P(n)=\lambda^{-1}[P(n-1)- \\
& \left.k(n) x(n)^{H} P(n-1)\right]
\end{aligned}
$$

Where $d(\mathrm{n})$ is the wanted signal of the RLS method and $x(n)$ is the input signal. $\mathrm{e}(\mathrm{n})$ is the a priori error, $k(n)$ is the gain vector, the same as $P(n) x(n), P(n)$ is the inverse of the ensemble-averaged autocorrelation matrix, $\lambda$ is the forgetting factor ${ }^{\text {ee }}$ of the RLS method.

\section{SIMULATION RESULTS USING ADAPTATION RLS ALGORITHM}

Computer simulations are used to drive the numerical results that are obtained here. The RLS-GSC algorithm performance is demonstrated using linear phased array of $N=$ 15 equally spaced elements. The distance between elements is half of the operation frequency related wavelength. Every antenna element has Seven delay components assigned to it $M=10$ and a range cell of interest was applied to (STAP) processor. $0 \mathrm{~dB}$ is the power of the target waveform which is used, relative to the noise at background and it has an angle of arrival $0^{\circ}$ degree referenced to the axis of the array. The target has a fixed Doppler frequency of $100 \mathrm{~Hz}$. Independent noises through taps of the CPI and the array elements were applied which has spectrum of a white Gaussian. A wideband jamming from Two sources represented at direction of arrival of $-20^{\circ}$ and $+50^{\circ}$ and $30 \mathrm{~dB}$ is the power of these jammers totally had also they had a full Doppler spectrum. Along with the jammers and noise, the array is impinged by many clutters from different DoAs. The clutters are between 180 and $-180^{\circ}$ and have a uniform distribution. These clutters has Doppler frequencies related to their DoAs

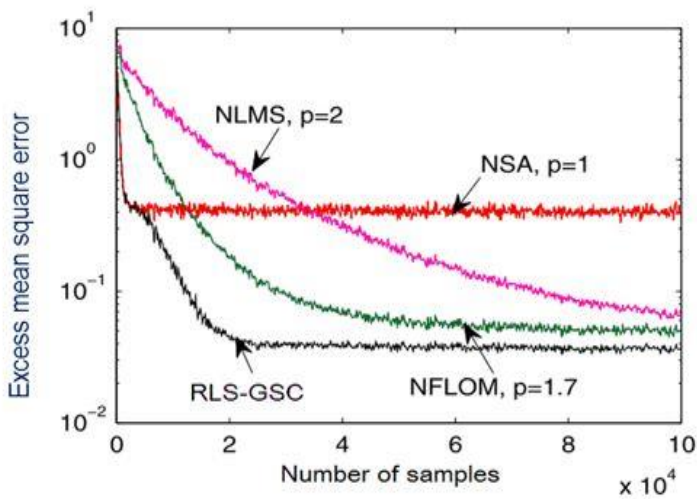

Fig. 12. The NFLOM and RLS-GSC approaches convergence curves in presence of background noises in addition to clutters and 2 wideband jammers (with total power30dBover the background noise)

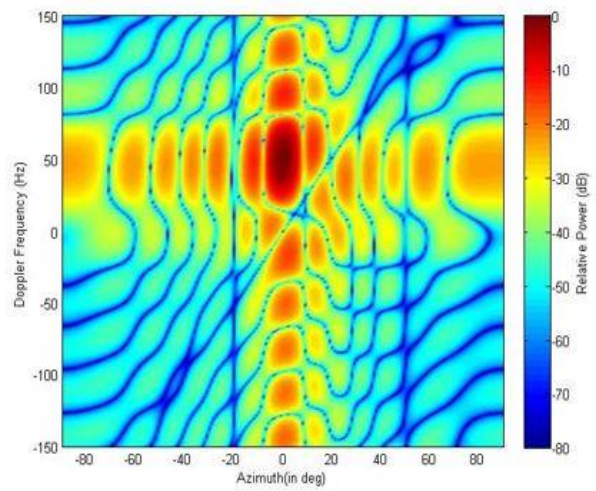

Fig. 13. Power Pattern for RLS-GSC Adaptive STAP of Target Azimuth and Doppler 


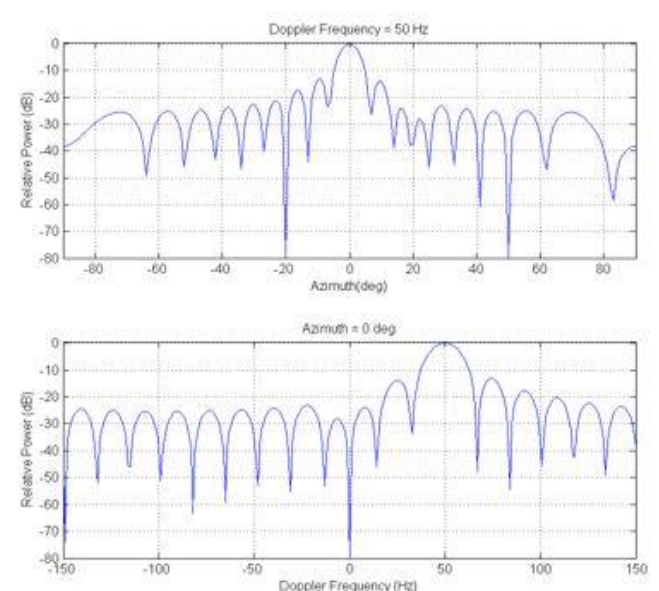

Fig. 14. RLS-GSC Principal Cuts at target of Doppler Frequency $=50 \mathrm{~Hz}$ and zero Azimuth angel

\section{XIV.CONCLUSION}

The adaptive filtering using the LMS algorithm at its simple structure delivers double the unknown parameters number of the scheme as a cost. Though, the algorithm has a rate of convergence heavily dependent on the spread of eigenvalue of the input correlation matrix. However, such a drawback is not suffered when the RLS-GSC algorithm is utilized but its complication of calculation is related to the squared of the unknown factors' number proportionally in the system.

As the real time processing has to be achieved when using STAP, the algorithms of minimum cost of computations must be engaged. The built NFLM-GSC structure provides a lower cost of adapted signal processing; therefore, it is closer for adaptive space-time processing task implementation at real time.

\section{REFERENCES}

[1] M.A. Richards, "Fundamentals of Radar Signal Processing". Chapter 9, McGraw-Hill, New York 2005.

[2] M.C. Wicks, et al., "Space-time adaptive processing: a knowledge-based perspective for airborne radar". IEEE Signal Process. Mag., vol. 23, 1, pp. 51-65, 2006.

[3] S.P. Applebaum and D.J. Chapman, "Adaptive Arrays with Main Beam Constarints". IEEE Trans. on Antennas and Propagation, vol. AP-24, 1976.

[4] L.J. Griffith and C.W. Jim, "An alternative approach to linearly constrained adaptive beamforming". IEEE Trans., vol. AP-30, pp. 27-34, Jan. 1982.

[5] S. Werner, J.A. Apolinario, and M.L.R.d. Campos, "On the Equivalence of RLS Implementations of LCMV and GSC Processors". IEEE Signal Processing Letters, vol. 10, no. 12, pp. 356-359 2003.

[6] A.M. Haimovich and Y. Bar-Ness, "An eigenanalysis interference canceller". IEEE Trans. On Signal processing, vol. 39, 1, pp. 76-84, Jan. 1991.

[7] I.S. Reed, J.D. Mallett, and L.E. Brennan, "Rapid Convergence Rate in Adaptive Arrays". IEEE Transactions on Aerospace and Electronic Systems, vol. AES-10, 6, pp. 853 - 863 Nov. 1974.

[8] I.P. Kirsteins and D.W. Tufts, "Adaptive detection using a low rank approximation to a data matrix". IEEE Trans. on Aerospace Electronic Systems, vol. AES-30, 1, pp. 55-67, Jan. 1994.
[9] R. Klemm, "Space-Time adaptive processing principles and applications". Inst of Engineering \& Technology (December 1, 1998).

[10] S. Haykin, "Adaptive Filter Theory". 4th edition. Chapters 5 and 6, Prentice Hall, New Jersey 2002.

[11] G. Tsoulos, "Smart Antennas for Mobile Communication Systems: Benefits and Challenges". IEE Electron. Commun Eng., vol. 11, 2, pp. 84-94, Apr. 1999.

[12] W. Liu and S. Weiss, "Wideband Beamforming: Concepts and Techniques". Wiley 2010.

[13] G. Strang, "Linear Algebra and its Applications". Harcourt Brace Jovanovich, San Diego, Ca 1988.

[14] G.H. Golub and C.F.V. Loan, "Matrix Computitions". The John Hopkins University Press, Baltimore, MD 1990.

[15] C.L. Koh, "Broadband adaptive beamforming with low complexity and frequency invariant response".Ph.D Thesis, University of Southampton, 2009.

[16] L. Maisel, "Performance of Sidelobe Blanking Systems". IEEE Transactions on Aerospace and Electronic Systems, vol. AES-4, 2, pp. 174 - 180, March. 1968.

[17] B.D.V. Veen, "Eigenstructure based partially adaptive array design". IEEE Transactions on Antennas and Propagation, vol. 36, 3, pp. 357-362, Mar. 1988.

[18] J.E. Hudson, "Adaptive array principles". Peter Peregrinus, London 1981.

[19] L.L. Scharf, "Statistical Signal Processing". Reading. Addison Wesley, MA 1991.

[20] P. Townsend, "Enhancements to the Generalized Sidelobe Canceller for Audio Beamforming in an Immersive Environment".Master Thesis, University of Kentucky, Kentucky, UK, 2009.

[21] Y.R. Zheng, G. Chen, and E. Blasch. "A normalized fractionally lower-order moment algorithm for space-time adaptive processing". in IEEE Conf. Military Communications, Orlando, FL, October 2007.

[22] P. Tsakalides and C.L. Nikias, "Robust space-time adaptive processing (STAP) in non-Gaussian clutter environments". IEE Proceedings - Radar, Sonar and Navigation, vol. 146, 2, pp. 8493, April. 1999. 